Aim To evaluate current clinical practice, with a focus on risk assessment at time of admission, and to compare this with the risk assessment framework proposed by the Royal College of Psychiatrists.

Methods All patients with an eating disorder requiring paediatric inpatient admission were identified over the period of June 2009 to February 2014. A retrospective casenote analysis was performed and data extracted using a standard proforma. Initial assessment of each patient was reviewed for documentation of BMI, weight, cardiovascular health (heart rate, syncope, significant orthostatic changes, irregular heart rate), ECG abnormalities, hydration status, temperature, biochemical abnormalities, disordered eating behaviours, engagement with management plan, activity and exercise, muscular weakness, self-harm/suicide, other mental health diagnoses as well as other potential co-existing risk factors.

Results A total of 14 patients were identified with 22 admissions over the data collection period. 15 patients were admitted electively via the local CAMHS team, 4 patients via A\&E and 3 patients following GP referral. No patients had a formal risk assessment performed. Assessment performed at the time of admission was highly variable. With the information available 10 patients were categorised as high risk, 11 patients were categorised as alert to high concern, and 1 admission was categorised as moderate risk. No patients were classified as low risk.

Conclusion This study highlighted the fact that children with eating disorders are treated in paediatric inpatient wards as well as in specialist centres. Admissions to the paediatric wards are infrequent. Initial assessment and investigation of this patient group is highly variable and does not adhere to current guidance. Implementation of a formal risk assessment framework is required in order to identify patients at risk of complications. The development of specific admission documentation based on Royal College guidance would aid assessment and help guide inpatient management, thereby providing a more consistent approach to patient care.

\section{G503(P) A 3 YEAR STUDY OF PAEDIATRIC MENTAL HEALTH ADMISSIONS TO A GENERAL PAEDIATRIC WARD}

N Johnson, H Massey, L White. Department of Paediatrics, Hinchingbrooke Hospital, Huntingdon, UK

\subsection{6/archdischild-2015-308599.456}

Aims The increasing prevalence of mental health problems is a well-recognised phenomenon in the paediatric population. While there are reviews of presentations to accident and emergency and to in-patient psychiatric hospitals, there are few studies of child mental health admissions to a general paediatric ward. The aim of this study was to investigate the prevalence of mental health conditions on a general medical paediatric ward and care pathways once admitted.

Method A retrospective case note study was performed of all cases admitted to a general paediatric ward between March 2011 and March 2014 in a district general hospital that required psychiatric review. Time of presentation, reason for presentation and admission, waiting time for psychiatric review, outcome of review and length of stay were all recorded, as well as the mental health background of those who presented.

Results 201 cases were identified, the youngest was 9 years old the median age was 15 years. Mental health admissions have increased from 38 between March 2011 and February 2012 to 93 from March 2013 to February 2014. The most common

\section{REASON FOR PRESENTATION}
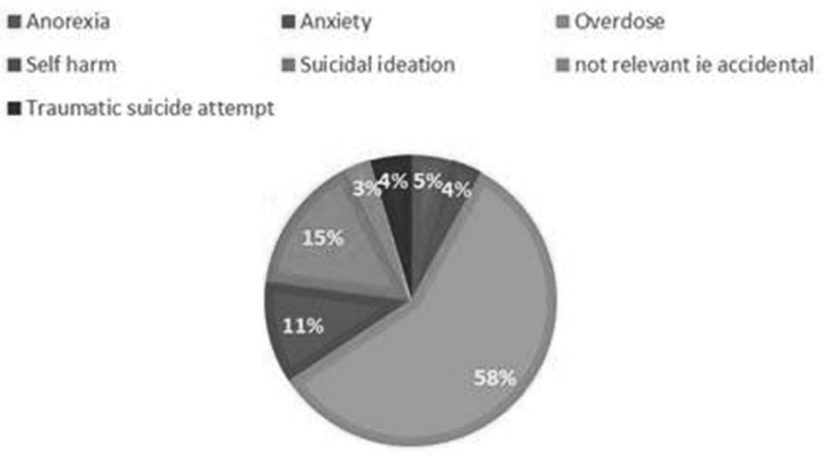

Abstract G503(P) Figure 1

reason for presentation (58\%) was overdose, followed by suicidal ideation (15\%) and self-harm (11\%), (Figure 1). Sixty percent of these admissions occur out of hours. $76 \%$ of children who present are already known to the Child And Adolescent Mental Health Service (CAMH). Of children admitted to the ward $49 \%$ required CAMH review only. Of these reviews, $16 \%$ occurred the same day as admission, $51 \%$ the following day and $6 \%$ waited more than a day for review. The most common outcome of review was discharge with CAMH follow up as an outpatient $(73 \%)$

Conclusion Mental health admissions to the general paediatric ward increased in our population, reflecting the national trend. Most of these admissions occurred out of hours when there is no specialist child mental health cover. As a consequence of this most children did not see a physician with expertise in mental health on the day of admission. Most admissions were discharged with ongoing mental health follow up adding to the burden placed on CAMH.

\section{G504(P) BELOW THE TIP OF THE ICEBERG - LIFE BEFORE TIER 3. A STUDY OF MENTAL HEALTH PROVISION IN SCHOOLS}

S Sayani, M Blair. Child Public Health Team, Northwick Park Hospital, Northwest London Hospitals NHS Trust, Harrow, UK

10.1136/archdischild-2015-308599.457

Background Due to lack of funding in the local borough, there is no formal Tier 2 clinical CAMHS (Child and Adolescent Mental Health Services). Tier 3 CAMHS will see children with severe mental health disorders, however those that don't meet the thresholds have to be supported by schools, children's services and charitable organisations.

Aims To find out what type of mental health support is provided in schools and to understand schools perspectives on this topic.

Method All 48 state schools in the local borough were emailed an electronic survey. The survey requested quantitative and qualitative responses. Qualitative responses were analysed thematically.

Results 21/48 (44\%) schools responded. 90\% had a counselling service but $42 \%$ of service providers did not have mental health training. The most common problem encountered by services was Anxiety and Depression (89\%). 53\% of schools expressed difficulties with the onward referral process to Tier 3 CAMHS. $21 \%$ of schools mentioned concern with the level of skills within their own service including lack of diagnostic abilities, as well as 
lack of supervision. $31 \%$ of schools felt more funding and provision was required in this area.

Limitations The data may be skewed because provision status in non-responders was unknown. The majority of non-responders $(81 \%)$ were primary schools. It is possible they did not respond because they did not have a service.

Conclusion The current mental health provision in schools cannot adequately substitute for a formal Tier 2 CAMHS service. Service provision is not uniform. Some schools had no service at all and some services did not meet Tier 2 criteria because providers were not mental health trained. Lack of skills within the service is worrying, particularly when dealing with depression, which needs adequate risk assessment. A difficult referral process to Tier 3 services may also be a barrier to accessing treatment. Considering the importance of early intervention to prevent significant mental health problems, the lack of good quality services at Tier 2 is concerning.

\section{G505(P) MEDICAL STUDENTS BUYING METHYLPHENIDATE ONLINE}

IJ Lane, ${ }^{2} \mathrm{~F}$ Finlay, ${ }^{2} \mathrm{H}$ Marcer. ${ }^{1}$ Bristol Medical School, University of Bristol, Bristol, UK; ${ }^{2}$ Child Health, Sirona, Bath, UK

\subsection{6/archdischild-2015-308599.458}

Background A study from US found that non-medical use of prescription stimulants in students ranged from $0-25 \%$, and a study from Cambridge University revealed that $10 \%$ of students have taken 'smart drugs.'

Although there are no studies in the UK on the prevalence of Methylphenidate use in medical students, we are aware of this practice first hand. Some report buying Methylphenidate online. Aims/Method We consulted colleagues in the legal profession, GMC guidelines, police and pharmacy to clarify implications of buying Methylphenidate online.

Results Methylphenidate and the law

Methylphenidate is a class B drug controlled drug.

It is unlawful to have a controlled drug in your possession unless you have authorisation in the form of a licence, eg. prescription.

The actual act of buying methylphenidate online is not illegal under the Misuse of Drugs Act but you commit an offence once you have the drug in your possession.

Possession could lead to up to 5 years in prison and an unlimited fine.

GMC Guidance The GMC has clear guidance on self-prescribing.

You must avoid prescribing for yourself or anyone with whom you have a close personal relationship.

You must not prescribe controlled medicines for yourself unless no other person with the legal right to prescribe is available to assess and prescribe without delay, which would put your life/health at risk, or cause unavoidable pain or distress.

Methylphenidate does not fit into this category. Students found to be self-prescribing could face a fitness to practice hearing/professional conduct meeting.

Conclusion There is anecdotal evidence that medical students in the UK are taking cognitive enhancement drugs.

The GMC has clear guidance on self prescribing - the offlicense use of Methylphenidate as a study aid would not be accepted.

Some are buying it from unregulated pharmacies online possession is illegal as Methylphenidate is a class B drug.
Students should be made aware of the risks so that they do not inadvertently compromise their health or future professional careers.

Universities should ensure that the issue of online purchasing and self medication, is explicitly discussed with medical students as part of their curriculum.

\section{G506(P) CRY A LITTLE TENDERNESS? DO DOCTORS CRY AND IS THIS ACCEPTABLE?}

EZA Bassett, F Finlay. Community Child Health, Sirona Health Care, Bath, UK

\subsection{6/archdischild-2015-308599.459}

Aims How common is it for doctors to cry? Do paediatricans cry as a sign of humanity and an expression of compassion? Is crying a weakness, a demonstration of incompetence? Or, in the right circumstance, does it enhance the doctor-patient relationship? A review of the literature will aim to answer these questions.

Methods Literature search

Results Doctors do cry. Sung ${ }^{1}$ found 69\% of medical students and $74 \%$ of interns cried at work. Wagner ${ }^{2}$ reported $57 \%$ of doctors, $31 \%$ of medical students and $76 \%$ of nurses cried. Women were more likely than men to cry. ${ }^{1,2}$ Medical students reported the highest negative social consequence of crying $^{2}$ stating it was often viewed as a sign of weakness. ${ }^{3}$ But views on crying are debated, ${ }^{4}$ opinions ranging from enthusiastic advocacy, guarded acceptance, ${ }^{5}$ outright rejection and condemnation. ${ }^{4}$

The concept of the detached physician ${ }^{6,7}$, remains alive and well. On the BMA Blog ${ }^{8}$ doctors debate learned behaviours to distance themselves from emotionally distressing situations. ${ }^{9}$ Others question if this is healthy ${ }^{4,8,9,10}$ debating if it is possible to have compassion, without emotion. The Francis Report $2013^{16}$ highlights the need for Compassion in Practice. ${ }^{17}$

Patients expect empathy and the demonstration of emotion by their doctor ${ }^{11,12}$ but only up to a point. ${ }^{13}$ Hojat $^{14}$ states it contributes to a better doctor-patient relationship with Anderson ${ }^{15}$ writing there is nothing wrong with a doctor who makes a patient feel nurtured while still being professional and appropriate.

Conclusion Humanity is the cornerstone of medicine. Depersonalization, with physicians distancing themselves from potentially emotionally challenging situations threatens the core principles of the profession. As paediatricians we need a balanced mix of rationality and compassion with an attitude of humility. We need to teach medical students how to break bad news, cope with baring witness to the dying process, and support those caught in the aftermath. We need to continue this education throughout medical careers while providing physicians with the resources to seek support as they need it.

\section{REFERENCES}

1 Sung $A D$, Collins ME, Smith AK, et al. Crying: experiences and attitudes of 3rd year medical students and interns. Teach Learn Med. 2009; 21(3):180-7

2 Wanger RE, Hexel M, Bauer WW, et al. Crying in hospital: a survey of doctors, nurses and medical students experience and attitudes. Med J Aust. 1997;166(1): 13-6

3 Angoff. A piece of my mind: crying in the curriculum. JAMA 2001; 286(9): 1017-8

4 Pruthi S, Goel A. Doctors do cry. Indian J Med Ethics 2014; 11(4):249-251

5 Majhail NS, Warlick ED. To cry or not to cry? physicians emotions at the bedside. Minn Med. 2011; 94(1):40-42

6 Sir William Osler, 1849 1919: A selection for medical students

7 Lief and Fox. Training for "detached concern" in medical students. The psychological basis of medical practice. New York: Harper and Row, 1963: 12-35 